\title{
Realistic propagation effects on wireless sensor networks for landslide management
}

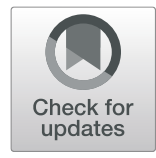

\author{
Nattakarn Shutimarrungson and Pongpisit Wuttidittachotti (iD
}

\begin{abstract}
This paper presents the development of propagation models for wireless sensor networks for landslide management systems. Measurements of path loss in potential areas of landslide occurrence in Thailand were set up. The effect of the vegetation and mountain terrain in the particular area was therefore taken into account regarding the measured path loss. The measurement was carried out with short-range transmission/reception at $2400 \mathrm{MHz}$ corresponding to IEEE 802.15.4 wireless sensor networks. The measurement setup was divided into two main cases, namely, the transmitting and receiving antennas installed on the ground and 1-m high above the ground. The measurement results are shown in this paper and used to develop propagation models suitable for operation of short-range wireless sensor networks of landslide management systems. The propagation model developed for the first case was achieved by fitting the averaged experimental data by the log-normal model plus the standard deviation. For the second case, the model was derived from the ray tracing theory. The mountain-side reflection path was added into the model which contained the reflection coefficient defined for the soil property. Furthermore, the resulting propagation models were employed in order to realistically evaluate the performance of wireless sensor networks via simulations which were conducted by using Castalia. In the simulations, the sensor nodes were placed as deterministic and random distributions within square simulated networks. The comparison between the results obtained from the deterministic and random distributions are discussed.
\end{abstract}

Keywords: Propagation models, Wireless sensor networks, Landslide management, Path loss measurement

\section{Introduction}

A landslide, which is a globally widespread and short-lived phenomenon, causes not only a number of human losses of life and injury but also extensive economic damage to private and public properties. The main factors of landslide occurrences are steep slope angles along with accumulated rainfall, moisture, and pore pressure saturation in the soil [1]. Thailand, located at the center of peninsular Southeast Asia and covered by a number of mountainous plateau areas, is one of the countries that most face rainfall-induced landslides every year [2]. In order to avoid or reduce the loss due to landslide disasters, there is a need for a landslide management system that can monitor and/or predict landslide occurrence.

A landslide management system is an essential key to reducing losses due to landslides by generating early

\footnotetext{
* Correspondence: pongpisit.w@it.kmutnb.ac.th

Faculty of Information Technology, King Mongkut's University of Technology North Bangkok, Bangkok, Thailand
}

warning for people living in potential landslide areas. In order to achieve an underlying system, sensors such as rain gauges, moisture sensors, piezometers, tiltmeters, geophones, and strain gauges can be installed in the potential landslide areas in order to collect the essential information needed to perform data analysis for landslide monitoring and prediction. Some examples of the use of sensors to monitor and/or predict landslides can be seen in $[1,3-6]$. Besides sensor technologies, a communication network is also required for sending the information collected by sensors.

Wireless sensor networks have received considerable interest in the research area of landslide monitoring and prediction, as seen in examples [1,5-7]. Another example was proposed to use a wireless sensor network in order to collect ambient data for general applications including landslide monitoring and prediction [8]. The performance of IEEE 802.15.4 commonly known as Zigbee, leading technology of short-range wireless sensor networks, was measured to verify that it can be used for 
a wide variety of applications [9]. In [10], the wireless sensor network was developed and then installed on the landslide area in Italy in order to monitor and manage the risks of landslides. Several parameters collected by sensors equipped with the coordinator of the wireless sensor network were employed to assess the possible risks and to provide useful information for an early warning system. Furthermore, an open-source wireless sensor system, called SMARTCONE, was designed and implemented for detection of the occurrence of the slope movement and debris flow of the hillside [11]. The performance of the proposed system was elevated via experimentation.

From the literature review, another interest is that the deployment of a wireless sensor network inside a potential landslide area requires knowledge of the node distance relevant to the path loss in order to provide full connectivity. An appropriate propagation model employed to predict such a path loss and the receivedsignal coverage is therefore essential for network planning. Extensive research has been conducted on propagation models, including theoretical and empirical models of wireless sensor networks. Some empirical models have been proposed in order to determine the path loss for a wide range of operating frequencies [1218]. Although these proposed models are simple, there is no parameter that controls the relationship between the models and the forest environments. In [19], a half-space model for dealing with wave propagation at the frequency of $1-100 \mathrm{MHz}$ in forest areas was proposed. In this approach, the associated phenomenon dominated by a lateral wave mode of propagation was also discussed. Subsequently, this approach was extended to the dissipative dielectric slab model in order to take the ground effect into account for the wave propagation at the frequency of $2-200 \mathrm{MHz}$ in the forest environment [20]. Recently, near-ground wave propagation was examined in a tropical plantation as seen in the example [21], where the experiment was conducted at very high frequency (VHF) and ultra-high frequency (UHF) bands. In this approach, the ITU-R model was slightly modified by taking the lateral wave effect into account. Moreover, the ITU-R model was further improved with considering the effect of the rain attenuation that was measured in Malaysia [22].

As mentioned, although those approaches are simple and valid for wave propagation in forest areas, they do not consider the realistic effects due to the environment in the context of landslide areas, especially in Thailand. In this paper, the measurement of path loss in one of the potential landslide areas in Thailand was examined. The measurement results were then employed to develop appropriate propagation models for particular landslide-monitoring/prediction applications. Simulations with realistic propagation effects were conducted in order to evaluate the performance of applying the wireless sensor network to the landslide management systems.

The main contributions of this paper are summarized as follows.

1. The measurement of the path loss was set up in accordance with the practical situation of applying the wireless sensor network to the landslide management of Thailand, whose climate and terrain are unique. In the measurement, the transmitting and receiving antennas of the short-range wireless sensor network at an operating frequency of 2400 $\mathrm{MHz}$ were placed on the ground and at a $1-\mathrm{m}$ height above the ground. This was done in order to investigate the effect of the antenna height on the propagation model.

2. The propagation models were then developed for the two cases, namely, the transmitting and receiving antennas installed on the ground and 1-m high above the ground. The first model was achieved by fitting the averaged experimental data by the developed model with the log-normal model plus the standard deviation. The second model was derived from the ray tracing theory. The mountain-side reflection path was added to the model which contained the reflection coefficient defined for the soil property.

3. The evaluation of the performance of the wireless sensor networks was presented via simulations, where the realistic wave propagation was taken into account.

Following this, the propagation prediction models are discussed along with the derivation of their equations in Section 2. These models are employed to compare with the model being proposed in this paper. The measurement and estimation of the path loss of the wireless sensor network are presented in Section 3. The simulation setup for evaluating the performance of the wireless sensor network for landslide management systems is discussed in Section 4, where their results and discussion are presented. Finally, the conclusions are drawn in Section 5. All results are discrbied by the data set of simulation and experimental results in Section 6 Additonal file 1.

\section{Basic principles of propagation prediction models}

In this section, we give an overview of the basic principles of propagation prediction models which were employed to compare with the proposed ones. There are several propagation models that have been employed to estimate path loss. One of the most simple and popular models is the free space loss as given by [23]: 


$$
\mathrm{PL}_{\text {free }}(\mathrm{dB})=-27.56+20 \log _{10}(f)+20 \log _{10}(d)
$$

where $f$ and $d$, respectively, are the frequency in megahertz and the distance between the isotropic transmitting and receiving antennas in meters. This theoretical propagation model is practicable for operation in the far-field region when there are no obstacles in the first ellipsoid of the Fresnel zone.

In [12], Weissberger developed a new empirical model that can estimate excess attenuation due to vegetation as written in the following:

$$
\mathrm{PL}_{\text {weissberger }}(\mathrm{dB})= \begin{cases}0.45 f^{0.284} d & d<14 \mathrm{~m} \\ 1.33 f^{0.284} d^{0.588} & 14 \mathrm{~m}<d \leq 400 \mathrm{~m}\end{cases}
$$

where $f$ and $d$ are in gigahertz and meters, respectively. The COST 235 models are proposed in [16] based on measurements conducted with a millimeter wave band between $9.6-57.6 \mathrm{GHz}$ through a grove of trees. The model divides the propagation scenario into two different conditions as follows:

$$
\operatorname{PL}_{\mathrm{COST}}(\mathrm{dB})= \begin{cases}15.6 f^{-0.009} d^{0.26} & \text { in leaf } \\ 26.6 f^{-0.2} d^{0.5} & \text { out of leaf }\end{cases}
$$

where $f$ and $d$ are in megahertz and meters, respectively. In [15], the FITU-R model was developed based on the ITU-R recommendation [13]. The optimization method for the numerical parameters using the least squared error that fit several sets of measurement data at the frequency of 11.2 and $20 \mathrm{GHz}$ was presented. The model was written as:

$$
\mathrm{PL}_{\text {FITU-R }}(\mathrm{dB})= \begin{cases}0.39 f^{0.39} d^{0.25} & \text { in leaf } \\ 0.37 f^{0.18} d^{0.59} & \text { out of leaf }\end{cases}
$$

where $f$ and $d$ are in megahertz and meters, respectively. The FITU-R model was also modified for the VHF and UHF bands from the measurements in a palm plantation at the frequency of $240 \mathrm{MHz}$ and $700 \mathrm{MHz}$. The modification takes the excess foliage loss with the lateral wave effect into account. This model is called a lateral ITU-R model [24], which can be used for long-range propagation in foliage areas and is defined by:

$$
\mathrm{PL}_{\text {LITU-R }}(\mathrm{dB})=0.48 f^{0.43} d^{0.13}
$$

where $f$ and $d$ are in megahertz and meters, respectively. This model is valid for in-leaf case.
In addition to the theoretical and empirical propagation models discussed above, a log-normal model, one of the most popular models based on the probabilistic distribution of the additional attenuation, has been widely used to predict path loss [25]. This model is defined by:

$$
\mathrm{PL}_{\text {log-normal model }}(\mathrm{dB})=\mathrm{PL}\left(d_{0}\right)+10 n \log _{10} \frac{d}{d_{0}}+X_{\sigma}
$$

where $n$ is the path loss exponent indicating the rate at which the signal attenuates with the distance. Generally, $n$ is equal to 2 for free space. $\operatorname{PL}\left(d_{0}\right)$ is the path loss at a known reference distance $d_{0}$ in the far-field region. $X_{\sigma}$ denotes a zero-mean Gaussian-distributed random variable (in $\mathrm{dB}$ ) with standard deviation $\sigma$ (in $\mathrm{dB}$ ). Experimentally, it has been found that the path loss in cluttered multipath environments is log-normally distributed, involving shadowing effects.

In order to guarantee the suitability of the models, the root means square error (RMSE) [15] between the data obtained from the predicted models and measurement should be determined. The RMSE is a useful tool that can be used to measure the difference between the path loss predicted by a model and measured by the radio frequency (RF) equipment. The RMSE is given by:

$$
\text { RMSE }=\sqrt{\frac{\sum_{i=1}^{k}\left(X_{\mathrm{obs}, i}-X_{\text {model }, i}\right)^{2}}{k}}
$$

where $X_{\text {obs }}$ and $X_{\text {model }}$ are measured and predicted data, while $k$ denotes the number of samples.

\section{Measurement and propagation estimation}

In this section, we discuss the setup of the measurement of the path loss. The measurement results are shown and then discussed as well. The propagation models suitable for the operation of wireless sensor networks of landslide management systems in a particular area of Thailand are introduced based on the existing models and our measurement results.

\subsection{Measurement setup}

In order to develop an appropriate propagation model for the short-range wireless sensor network of the landslide management systems, measurements were conducted in a potential landslide area on the small mountain of Nakhon Ratchasima province in Thailand during the rainy season, when the average monthly rainfall was $80 \mathrm{~mm}$. The area chosen for the measurement was similar to a bald mountain where the terrain mainly consists of soil and sand. Figure 1 shows a basic block diagram of our measurement setup. The RF propagation 


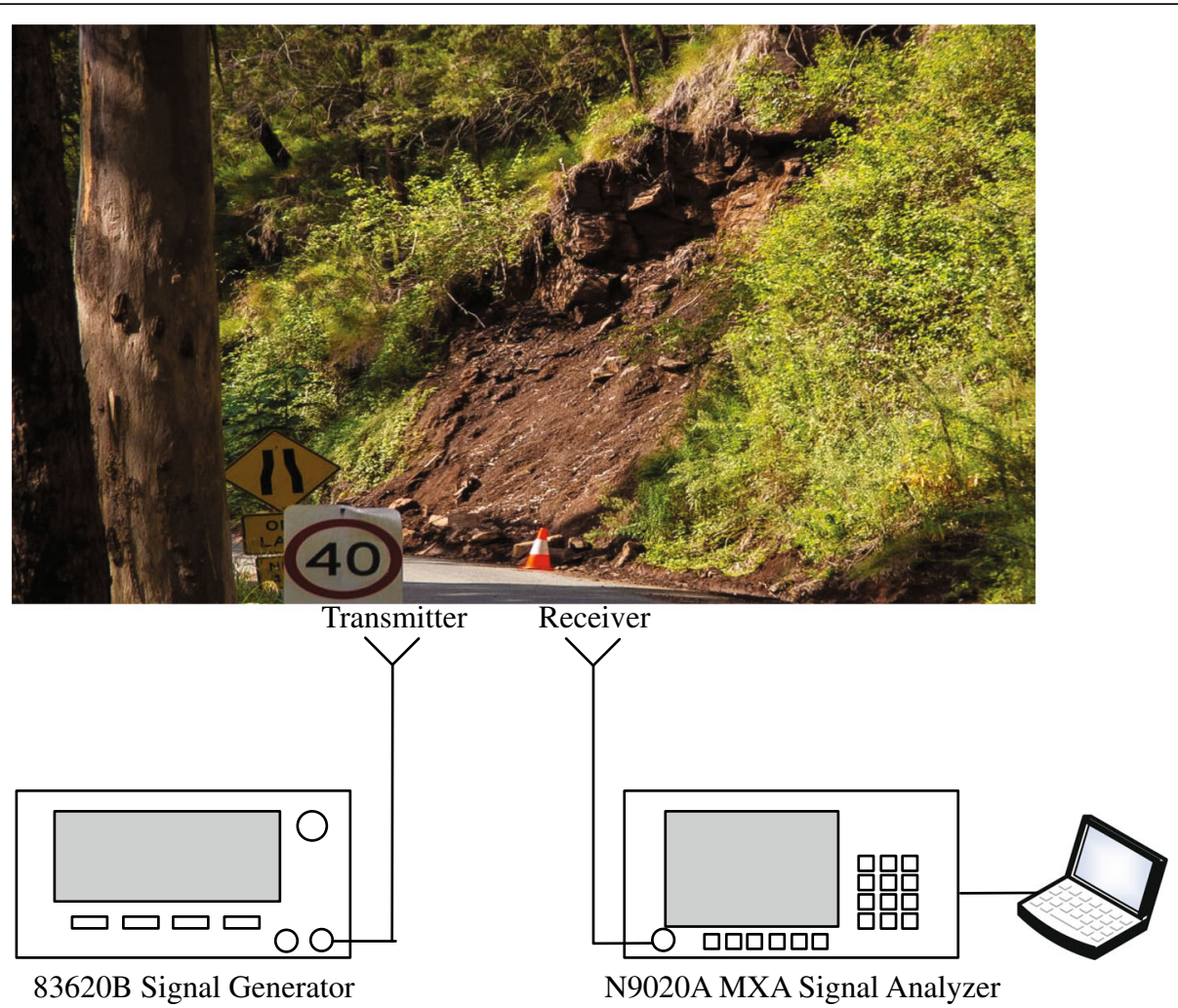

Fig. 1 Basic block diagram of the measurement setup

measurements were performed at $2400 \mathrm{MHz}$ by using RF equipment, including a signal generator (83620B Hewlett Packard), a spectrum analyzer (N9020A MXA Agilent technologies), and transmitting and receiving antennas. The continuous wave $(\mathrm{CW})$ was generated by using a signal generator at $2400 \mathrm{MHz}$ with the power of $17 \mathrm{dBm}$. The vertically polarized omnidirectional antennas with a typical gain of $5 \mathrm{dBi}$ were employed for the measurement. The loss in the RF cable was $5.6 \mathrm{~dB}$. The measurement data were captured by using a spectrum analyzer and stored into a control computer via GPIB interface for post-processing. In this paper, the measurement was divided into two different main cases. First, the transmitting and receiving antennas were placed on the ground since it is easy to place many small sensors, including transmitters and receivers, on ground in order to measure the data, such as seismic vibrations, average monthly rainfall, and relative humidity, which are used for landslide detection in the practical situation of wireless sensors networks. Second, the height of the receiving and transmitting antennas was $1 \mathrm{~m}$ above the ground. This was done in order to determine the effect of the antenna height on the propagation model. In some scenarios, a $1-\mathrm{m}$ antenna tower can be probably installed in the landslide area. Figure 2 shows the RF-propagation measurements done in the potential landslide area. In order to study the short-range wave propagation in such an area, the distance $d$ between the transmitting and receiving antennas was varied from 0.5 to $50 \mathrm{~m}$ with a step of $0.5 \mathrm{~m}$, resulting in 100 measured positions. In an individual position, the measurement was repeated 30 times in order to achieve accurate results. The system calibration of the measurement data was performed by the removal of the antenna gain and the cable loss of the transmitter and receiver. Note that our measurement was done horizontally on the mountain. The vertical direction was no longer under consideration because of the limitation of measurement setup. However, this problem will be resolved and discussed in a future publication.

\subsection{Measurement results and developed propagation models}

In order to evaluate the possibility of the use of the well-known propagation models, including COST235, FITU, LITU-R, Weissberger, log-normal, and free space models, to achieve the appropriate path-loss prediction in the landslide area, the measured path loss versus the distance for the first case of the antennas on the ground were plotted, together with its average and COST235, FITU, LITU-R, Weissberger, log-normal, and free space models, as shown in Fig. 3. In the figure, the ability to 

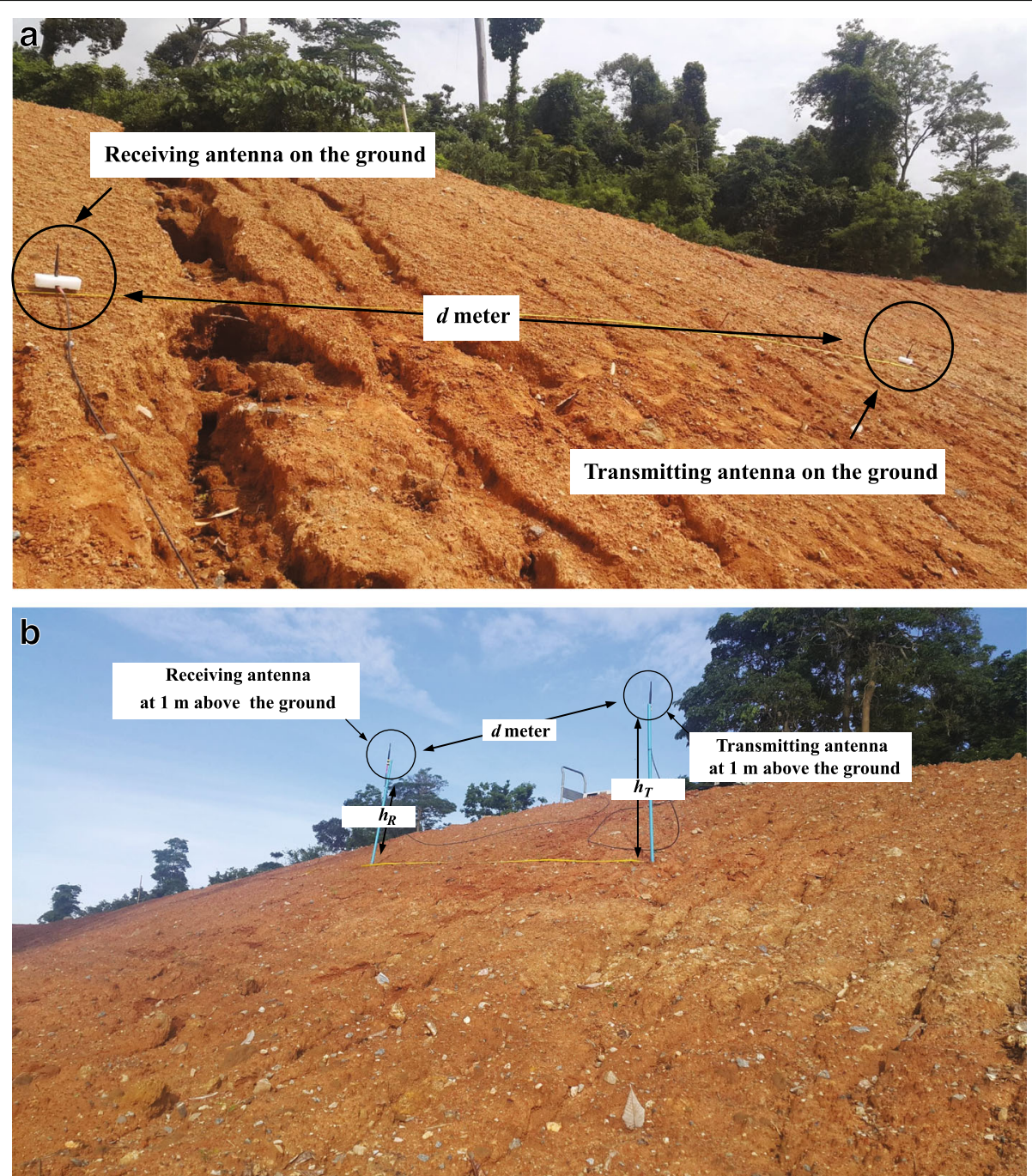

Fig. 2 Measurement setup. a First case. b Second case

predict the path loss using COST235, FITU, LITU-R, Weissberger, and free space models becomes poor when the distance increased. These models underestimated the path loss significantly by up to $68 \mathrm{~dB}$ at $25 \mathrm{~m}$ under the measured data. On the other hand, the log-normal model, whose path loss exponent was initially deduced as $n=2.4$, was more suitable to be used for curve fitting. The path loss exponent was then varied as $n=1.5,1.6$, 1.7, 1.8, and 1.9. Figure 4a shows the path loss of the log-normal model with varying the path loss exponent. The root mean square (RMS) error given in (7), between the measured data and path loss predicted by the log-normal model, was calculated in order to investigate the performance of the fitted curve. The RMS errors obtained from the log-normal models with $n=1.5,1.6,1.7$, 1.8 , and 1.9 were $3.16,2.63,3.02,4.08$, and 5.43 , respectively. It can be seen that the path loss of the log-normal model with $n=1.6$ was closest to that of the averaged measurement data. In this paper, we developed the propagation model from our measurement results of wireless sensor networks, specifically for landslide management systems. Reconsidering the measurement results, the measured path loss at $1 \mathrm{~m}$ was $52.53 \mathrm{~dB}$ and was then chosen as the reference distance path loss $\operatorname{PL}\left(d_{0}\right)$. Thus, the propagation model developed for the wireless sensor network for the landslide management systems was achieved on the basis of the log-normal model and our measurement data as given by:

$$
\mathrm{PL}_{\text {on ground }}(\mathrm{dB})=\mathrm{PL}\left(d_{0}\right)+10 n \log _{10} d+\mathrm{SD}
$$

where SD denotes the measured standard deviation of the received power around the average. Here, the standard deviation SD of this measurement case was 8.42. The path loss $\mathrm{PL}\left(d_{0}\right)$ at a known reference distance $d_{0}=$ 


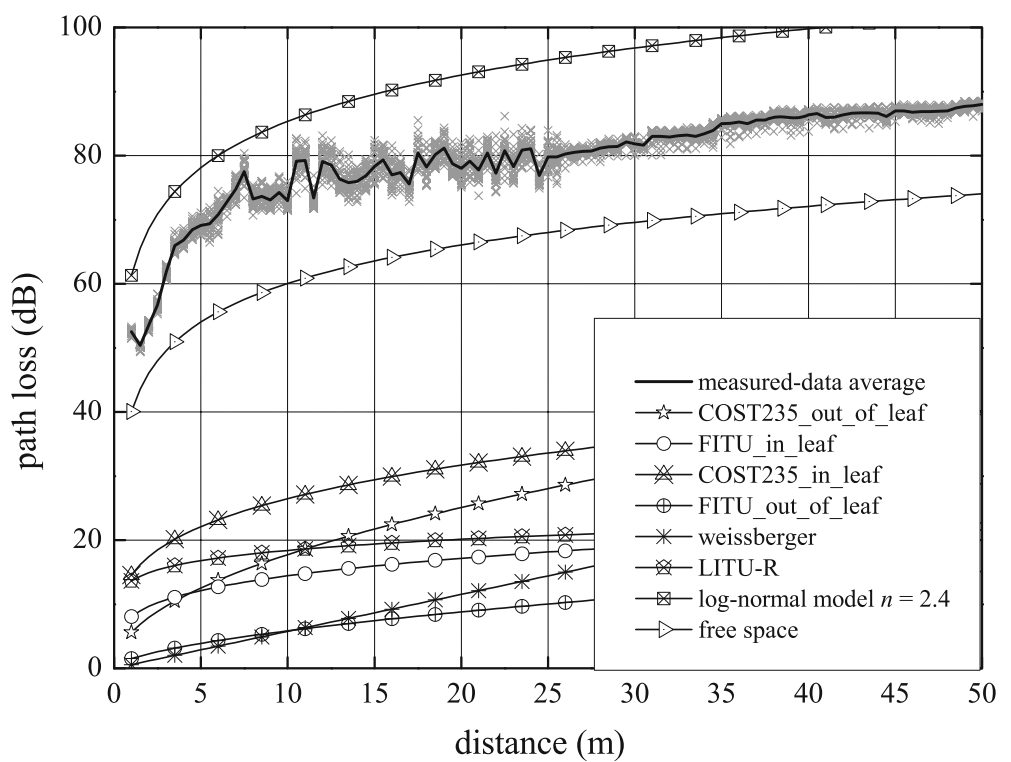

Fig. 3 Path loss obtained from measurement and prediction of the first case with antennas on the ground

$1 \mathrm{~m}$ was $52.53 \mathrm{~dB}$. Figure $4 \mathrm{~b}$ shows the path loss of the developed propagation model with varying its path loss exponent as $n=1.4,1.5,1.6,1.7,1.8,1.9$, and 2 . The RMS errors between the developed propagation model and averaged measurement data were $3.20,3.07,3.48$, $4.30,5.33,6.47$, and 7.68 when $n=1.4,1.5,1.6,1.7,1.8$, 1.9 , and 2 , respectively. Note that the path loss of the developed propagation model with $\mathrm{SD}=8.42$ and $n=1.5$ was almost identical to that of the predicted conventional log-normal model. This implies that we can use our developed propagation model instead of the conventional log-normal model for the wireless sensor network for the landslide management systems.

Figure 5 shows the predicted and measured path losses obtained from the second case of a 1-m antenna height above the ground. In the figure, the log-normal and free space models were employed to predict the path loss. It was seen that the log-normal models with different path loss exponents, i.e., $n=1.8,2.0$, and 2.4, overestimated the measured path loss significantly. The RMS errors of the use of the log-normal models with $n=1.8,2.0$, and 2.4 were $8.50,11.42$, and 17.63 , respectively. On the other hand, the RMS error of the use of the free space model was 3.21. Although the use of this model to predict the measured path loss was more suitable than using the log-normal model, the RMS error was somewhat high as compared with the former case. Note that there were some significant fluctuations which appeared at the distance 21 to $26 \mathrm{~m}$ of the measured data. A propagation model specific for the wireless sensor network of the landslide management systems should be considered when employing 1-m-high antennas above the ground. In this paper, we introduced the development of the propagation model by using a multiple-ray tracing model to fit the measured data.

In case of the 1-m-high antennas, we determined a basic geometry model for the measurement setup again, as depicted in Fig. 6. The transmitting and receiving antennas were placed on the mountain at heights of $h_{T}$ and $h_{R}$, respectively. The $d$ denotes the direct distance between transmitting and receiving antennas. In this paper, the propagation model developed by using the multi-ray tracing considers three major coexisting transmission paths, namely, line-of-sight (LOS), ground reflection, and mountain-side reflection paths. Note that the mountain-side reflection path was added to the developed model instead of the conventional two-ray ground-reflection model. The total received power was calculated from the combination of the individual received power from LOS, ground reflection, and mountain-side reflection paths. Based on the Friis transmission formula, we derived the equation of the received power from three-ray tracing including line-of-sight (LOS), ground reflection, and mountain-side reflection paths, as written by:

$$
\begin{array}{r}
P_{r}=P_{t}\left(\frac{\lambda}{4 \pi}\right)^{2}\left(\frac{\sqrt{G_{\mathrm{LOS}}}}{d_{\mathrm{LOS}}}-\Gamma_{\mathrm{gr}} \frac{\sqrt{G_{\mathrm{gr}}} e^{-j 2 \pi\left(d_{\mathrm{gr}}-d_{\mathrm{LOS}}\right) / \lambda}}{d_{\mathrm{gr}}}\right. \\
\left.-\Gamma_{m} \frac{\sqrt{G_{m}} e^{-j 2 \pi\left(d_{m}-d_{\mathrm{LOS}}\right) / \lambda}}{d_{m}}\right)^{2}
\end{array}
$$

where $P_{r}$ and $P_{t}$ denote the received and transmitted powers, respectively. $G_{\mathrm{LOS}}, G_{\mathrm{gr}}$ and $G_{m}$ are the 


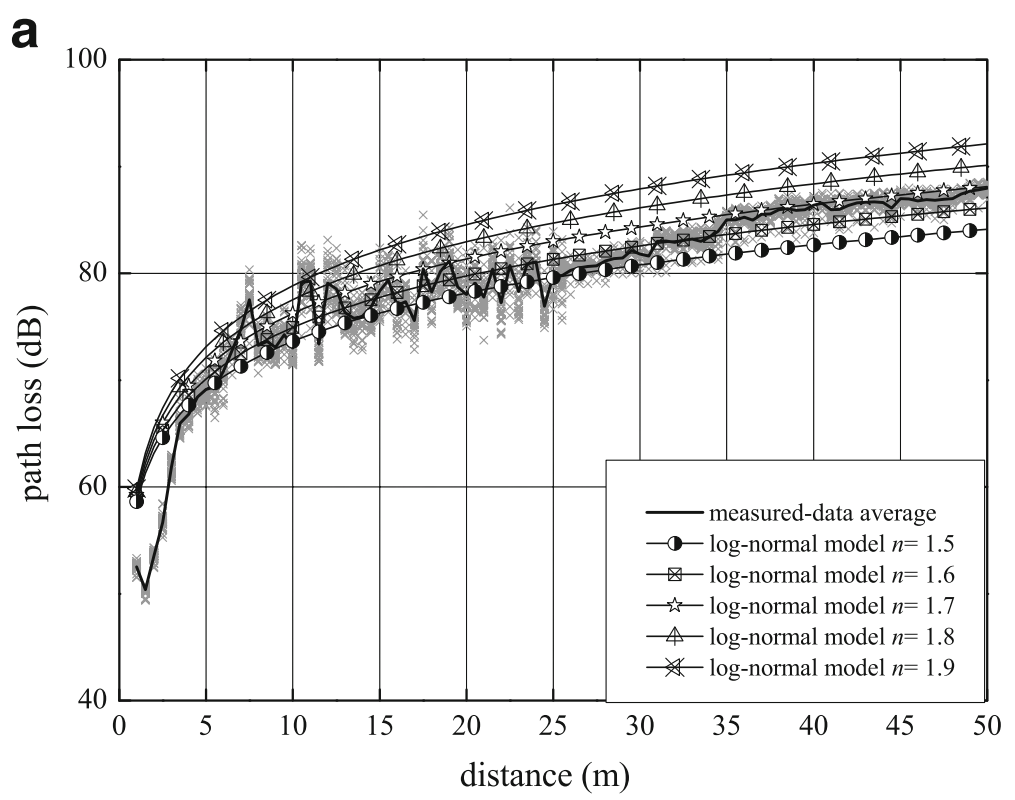

b

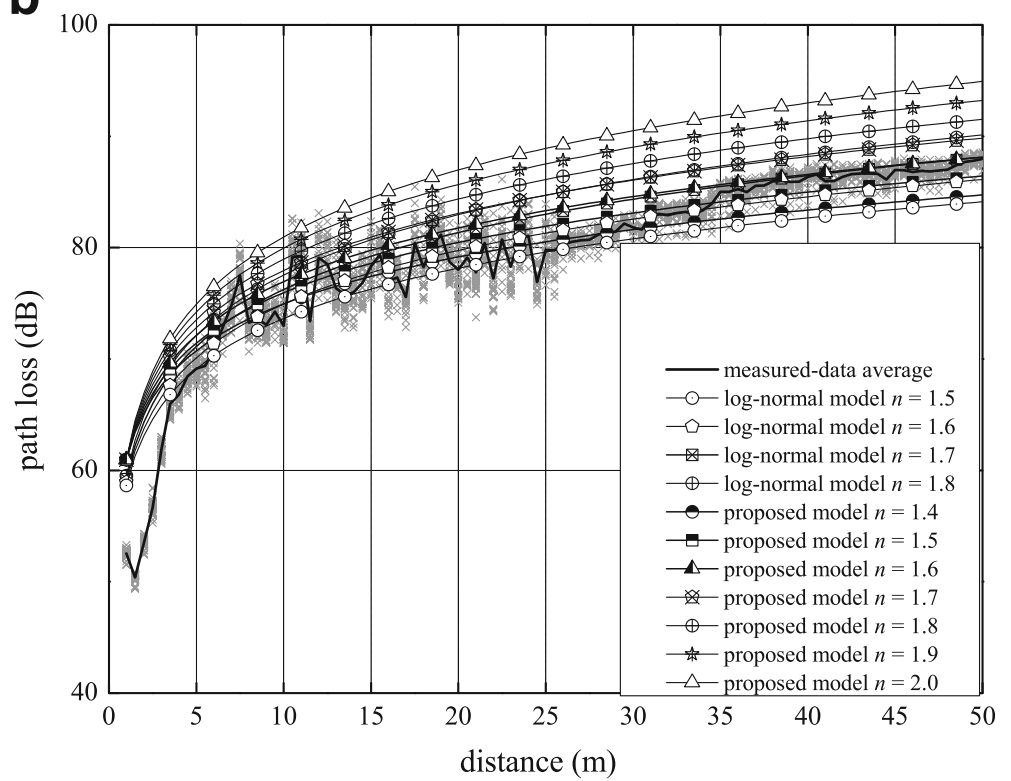

Fig. 4 Path loss of a log-normal model and $\mathbf{b}$ developed propagation model, compared with measurement data of the first case with antennas on the ground

combined antenna gains along the LOS, ground reflection, and mountain-side reflection paths. Since the pattern of all antennas employed in our measurement was omnidirectional, the $G_{\mathrm{LOS}}, G_{\mathrm{gr}}$ and $G_{m}$ were therefore equal. The $\Gamma_{\mathrm{gr}}$ and $\Gamma_{m}$ were reflection coefficients of the ground and mountain side, respectively. Since the measurement setup was conducted on a mountain, the $\Gamma_{\mathrm{gr}}$ and $\Gamma_{m}$ were equal as well, which were re-denoted as $\Gamma$. Practically, the reflection coefficient $\Gamma$ can be achieved by measurement of the soil property. The use of the reflection coefficient for path loss prediction is very useful when there is a need to change the considered landslide area having different soil properties. The distances of the wave travel from the transmitting antenna to the receiving antennas along the LOS, ground reflection, and mountain-side reflection paths were denoted as $d_{\mathrm{LOS}}, d_{\mathrm{gr}}$ and $d_{m}$, respectively. Applying trigonometry to this problem, the distances $d_{\mathrm{LOS}}, d_{\mathrm{gr}}$ and $d_{m}$ can be given by: 


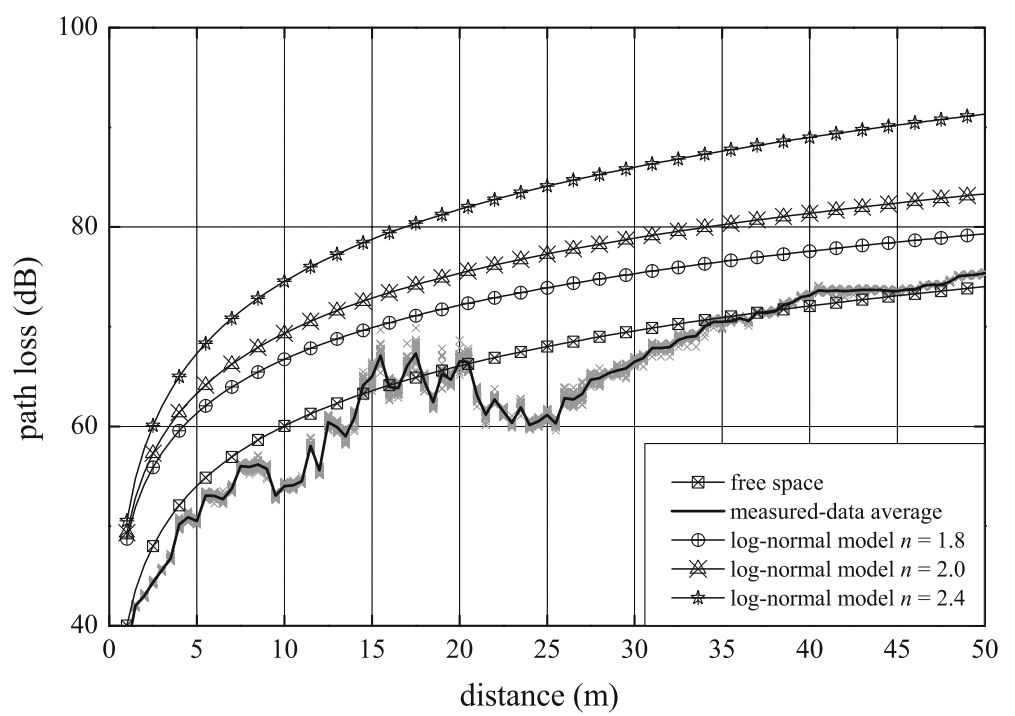

Fig. 5 Path loss obtained from measurement and prediction of the second case with 1-m antenna height above the ground

$$
\begin{gathered}
d_{\mathrm{LOS}}=\sqrt{\left(h_{T}-h_{R}\right)^{2}+d^{2}} \\
d_{\mathrm{gr}}=\sqrt{\left(h_{T}+h_{R}\right)^{2}+d^{2}}
\end{gathered}
$$

and

$$
d_{m}=\sqrt{\left(h_{T} \cot \theta+h_{R} \cot \theta\right)^{2}+(d / 2)^{2}}
$$

These equations indicate that the distances $d_{\mathrm{LOS}}, d_{\mathrm{gp}}$ and $d_{m}$ depended upon the antenna height, mountain slope $\theta$, and direct distance $d$ between the transmitting and receiving antennas.

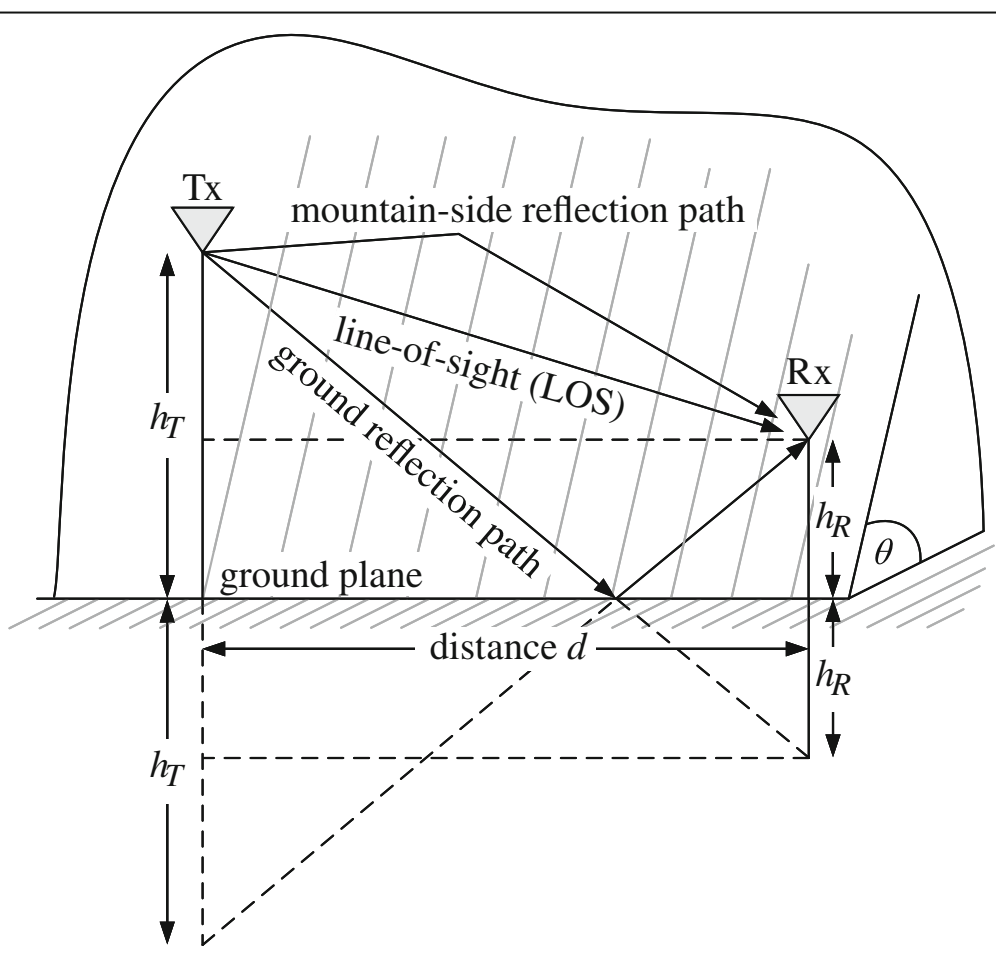

Fig. 6 The basic geometry of the experimentation setup on the mountain in the second case of 1-m-high antennas 
To simplify (9) and to fit the experimental data with the path loss $\mathrm{P}_{\mathrm{L}}\left(d_{0}\right)$ at a known reference distance $d_{0}$, the total path loss can be rewritten as

$$
\begin{aligned}
& \mathrm{PL}_{\text {above ground }}(\mathrm{dB})=\operatorname{PL}\left(d_{0}\right) \\
& \quad+20 \log \left[\frac{1}{d_{\mathrm{LOS}}}-\Gamma \frac{e^{-j 2 \pi\left(d_{\mathrm{gr}}-d_{\mathrm{LOS}}\right) / \lambda}}{d_{\mathrm{gr}}}-\Gamma \frac{e^{-j 2 \pi\left(d_{m}-d_{\mathrm{LOS}}\right) / \lambda}}{d_{m}}\right]
\end{aligned}
$$

Here, the path loss $\mathrm{P}_{\mathrm{L}}\left(d_{0}\right)$ at a known reference distance $d_{0}=1 \mathrm{~m}$ is $37.54 \mathrm{~dB}$. Since the short distances $d_{\mathrm{LOS}}, d_{\mathrm{gr}}$ and $d_{m}$ were not approximately equal because the determined distance was short.

Figure 7 shows the path loss of the developed propagation model based on the multi-ray tracing, compared with that of the free space model. Here, the slope of the mountain was set as $\theta=30^{\circ}$. The reflection coefficient $\Gamma$, which mainly depends upon the soil property, was varied in order to investigate the appropriation of the developed propagation model. $\Gamma=-1$ indicates that the soil reflects all of the transmitted power to the receiving antennas. The RMS errors obtained from the developed propagation model were $3.19,4.48,2.94$, and 3.22 when $\Gamma=0,-0.4,-0.8$, and -1 , respectively. The developed propagation model based the multi-ray tracing and our measurement data achieved the smallest RMS error when $\Gamma=-0.8$. Moreover, it should be noted that the path loss obtained from the developed propagation model when $\Gamma=0$ which means the transmitted power was completely absorbed by the soil, was almost identical to that of the free space model. This indicates that there was no reflection from the ground and mountain-side paths. There exists only the LOS path in the path loss of the predicted results. This also reveals that our developed propagation model can be applied to other mountains that possess different soil properties by choosing the appropriate reflection coefficient $\Gamma$.

\section{Practical simulations for wireless sensor networks}

\subsection{Simulation scenario}

Simulations were conducted using Castalia, an extension of OMNET++, in order to evaluate the performance of the wireless sensor networks for landslide management systems. In our simulations, the measurement results presented in the previous section were employed instead of the default radio model. The realistic propagation effects on the wireless sensor networks were therefore taken into account. The IEEE 802.15.4 standard was chosen as Physical (PHY) and Medium Access Control (MAC) layers with an operating frequency of $2400 \mathrm{MHz}$, corresponding to that of our measurement setup. The routing protocol deployed for the wireless sensor networks of the landslide management systems was an Ad hoc On-Demand Distance Vector (AODV). The simulations were run at a fixed duration of $500 \mathrm{~s}$ with a packet generated at a constant packet rate of 0.5 packets per second. The wireless channel bit rate was $250 \mathrm{kbps}$. All sensor nodes placed in the simulations were configured to set their transmission power at $57.42 \mathrm{~mW}$ and their sensitivity value at $-100 \mathrm{dBm}$ based on the Chipcon CC2420 transceiver chip. Omnidirectional antennas with a typical gain of $5 \mathrm{dBi}$ were employed for signal transmission and reception.

The simulations were set up corresponding to the practical situation in the potential landslide area of

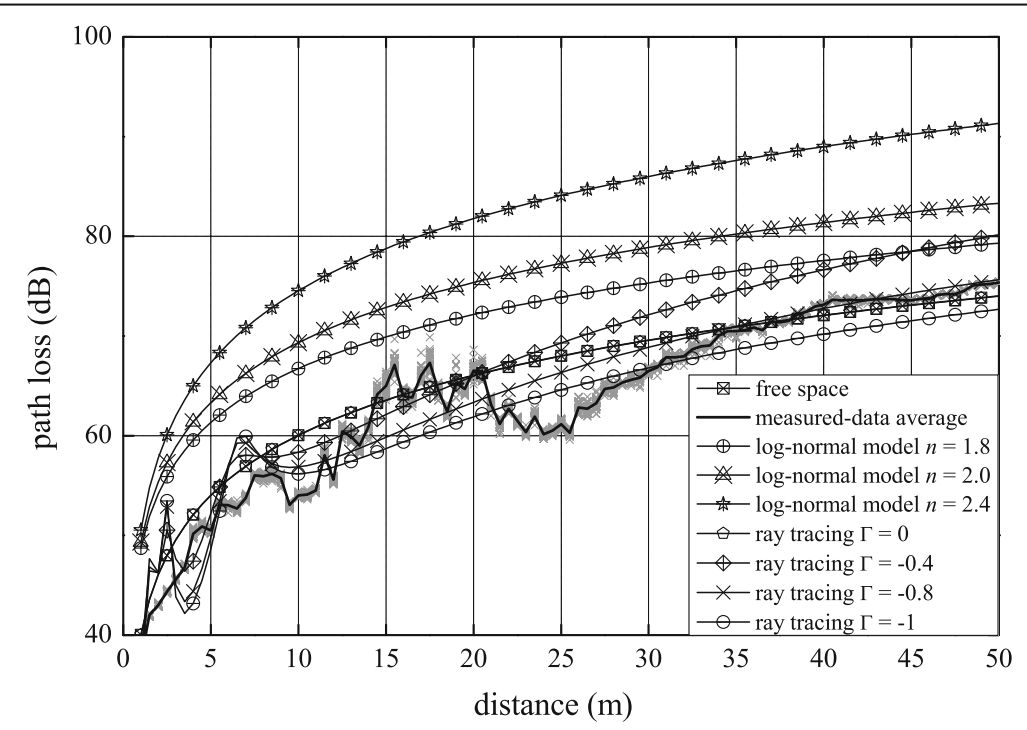

Fig. 7 Path loss of the free space model and developed propagation model of the second case with 1-m antenna height above the ground 
Thailand. Simulations were distinguished into two main catalogs in accordance with the measurement cases. First, the path loss obtained from the measurement with the antennas placed on the ground was employed as a radio model in the simulations in order to investigate the realistic propagation effects due to the antenna position and other environmental parameters. Second, we employed the measurement results of the case of 1-m-high antennas above the ground in order to demonstrate the effect of the antenna height on the performance of the wireless sensor networks.

Figure 8 shows the network simulation models. In each catalog of simulations, the node positions of the simulated networks were deterministically and randomly distributed

a

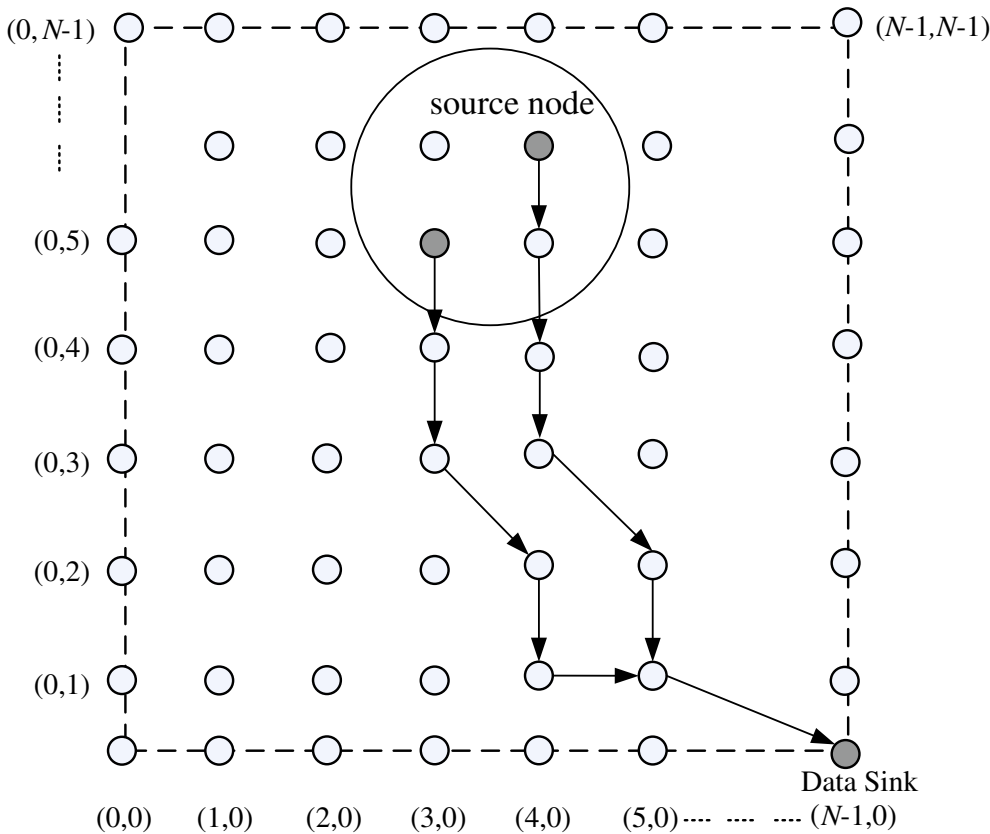

b

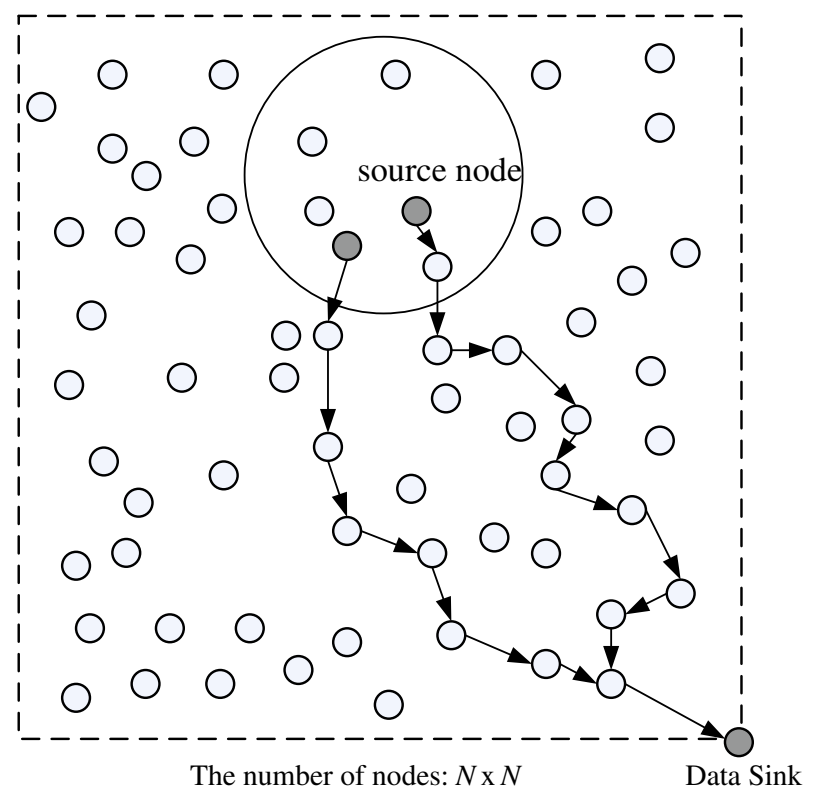

Fig. 8 Network simulation models with a deterministic and $\mathbf{b}$ random distributions 
within a $300 \mathrm{~m} \times 300 \mathrm{~m}$ square as shown in Fig. $8 \mathrm{a}$ and b, respectively. In practical situations, sensors installed to predict a landslide occurrence are probably deployed either randomly or deterministically depending on the selection of the user. Thus, the deterministic and random distribution of the node placement should be determined. This simulation consisted of $N \times N$ sensor nodes. The distance between two adjacent sensor nodes in our simulation model depended upon the number of placed sensor nodes. All of the sensor nodes were stationary, and the sink was located at the bottom right of the network area as shown in the figure. It should be noted that the sink can send information gathered by the sensor nodes to the gateway node, which may be installed outside the potential landslide area. The gathered data received from the gateway node through communication networks such as Internet or mobile networks can be used to calculate/predict the landslide occurrence at the monitoring center.

\subsection{Results}

In order to evaluate the performance of the wireless sensor networks for the landslide management systems, the

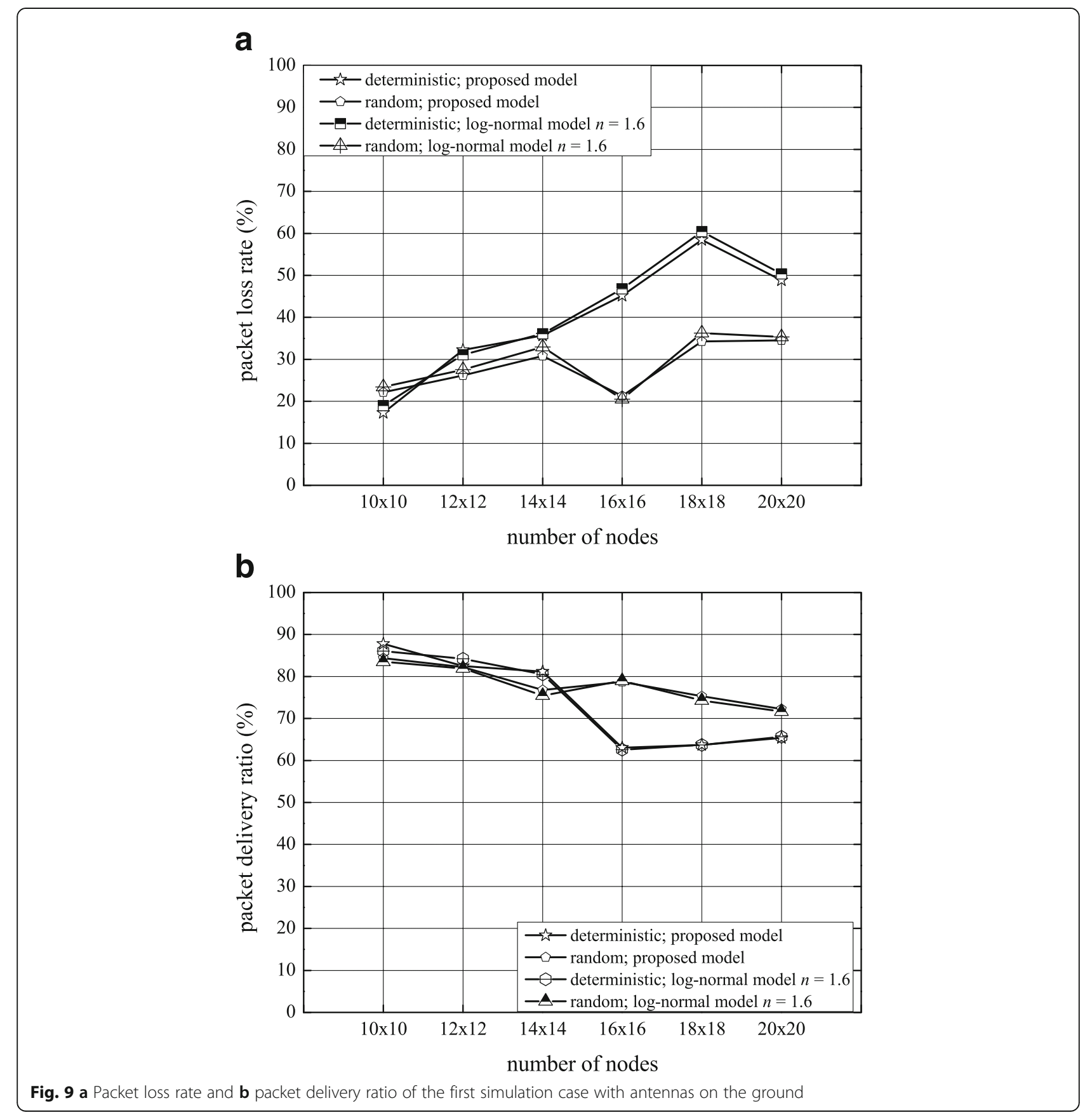


matrices, that are the packet loss rate and packet delivery ratio, are determined in this section. The number of sensor nodes was varied from $10 \times 10$ to $20 \times 20$. Figure 9 shows the packet loss rate and packet delivery ratio versus the number of sensor nodes when the antennas were placed on the ground. It was seen that the packet loss rate increased proportionally to the number of nodes. The collision probably occurs when the density of nodes is high. The packet loss rate slightly decreased at $N=$ $20 \times 20$ since the probability of success of finding a communication path was high. The packet delivery ratio decreased when the number of nodes increased. Although the packet loss rate suddenly decreased when $N$ $=16 \times 16$, compared with that when $N=15 \times 15$, the packet delivery ratio still was high. In the case of the deterministic node distribution, the distance between the two neighbor nodes of $N=16 \times 16$ and $N=14 \times 14$ was 23 and $20 \mathrm{~m}$, respectively. The packet loss rate of the case of the random node distribution was less than $40 \%$ for all node numbers under consideration while that of the deterministic node distribution was greater than $40 \%$ for $N=16 \times 16$ to $N=20 \times 20$. On the other hand, the

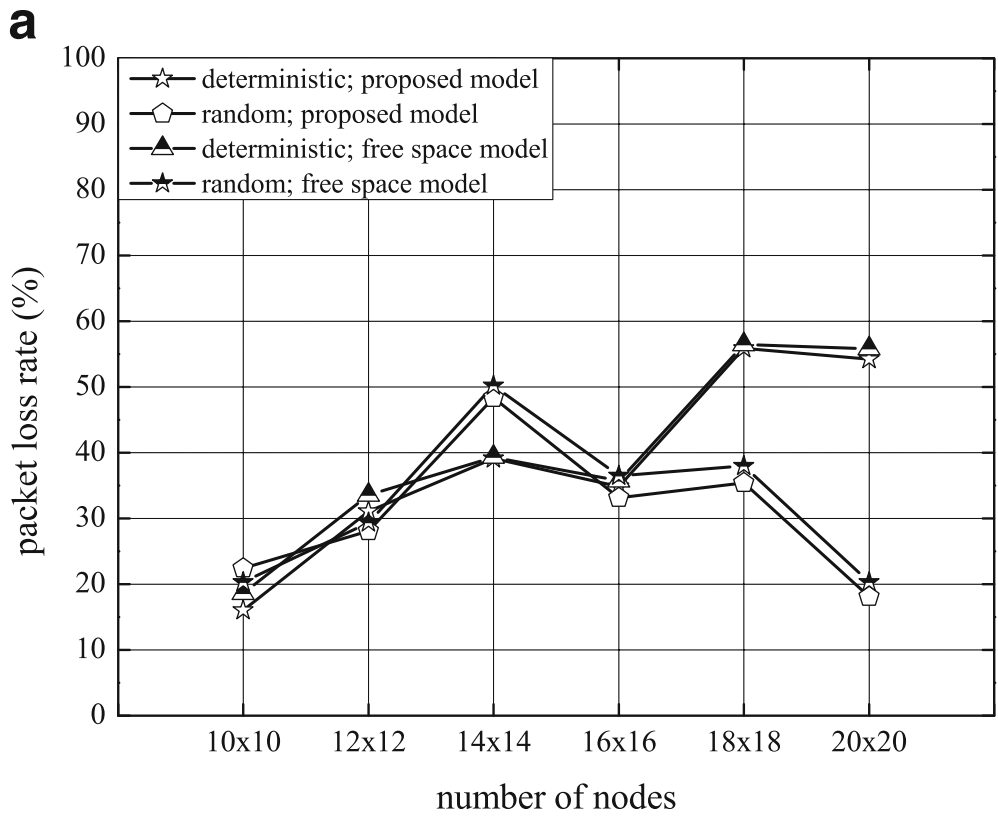

b

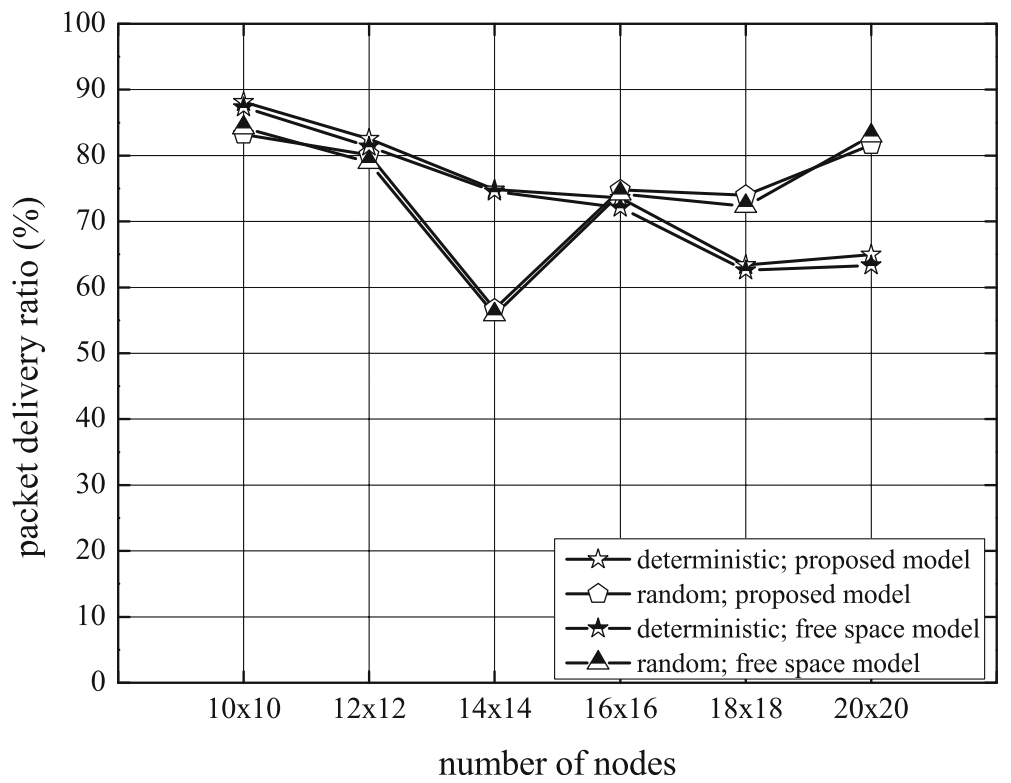

Fig. 10 a Packet loss rate and $\mathbf{b}$ packet delivery ratio of the second simulation case with 1-m-high antennas above the ground 
packet delivery ratio of the random node distribution was greater than $70 \%$ of all node numbers under consideration while that of the deterministic node distribution was less than $70 \%$ for $N=16 \times 16$ to $N=20 \times 20$. Note that simulation results obtained from the use of the proposed model and the log-normal model were almost identical.

Figure 10 shows the packet loss rate and packet delivery ratio versus the number of sensor nodes when the antennas were placed at a 1-m height above the ground. The packet loss rate and packet delivery ratio of this case were similar to those when the antenna was placed on the ground. The random node distribution had a slightly better packet loss rate and packet delivery ratio than the deterministic node distribution. The simulation results obtained from the use of the proposed model and free space model were almost identical as well.

\section{Conclusion}

In this paper, the development of propagation models has been proposed for wireless sensor networks for landslide management systems. The propagation models were developed based on the measurement data and existing propagation models. For the development, the measurement was set up using two main cases-transmitting and receiving antennas installed on the ground and 1-m high above the ground. The propagation models for the first and second scenarios were developed based on the log-normal model and multi-ray tracing models along with our experimental data, respectively. The path loss versus distance was shown to validate that the developed propagation models were suitable for the operation of landslide management systems using a wireless sensor network. Furthermore, the resulting propagation models were employed in order to realistically evaluate the performance of wireless sensor networks via simulations, which were conducted using Castalia. In the simulations, the sensor nodes were placed as deterministic and random distributions. The simulation results have been shown to confirm that the short-range wireless sensor network at an operating frequency of $2400 \mathrm{MHz}$ can be employed for landslide management systems.

\section{Additional file}

Additional file 1: Data set of simulation and experimental results. (XLSX $60 \mathrm{~kb})$

\section{Abbreviations}

AODV: Ad hoc on Demand Distance Vector; FITU: Fitted ITU; IEEE: The Institute of Electrical and Electronics Engineers; ITU-R: The International Telecommunication Union-Radiocommunications Sector; LITU-R: Lateral ITU; MAC: Media access control; PHY: Physical layer; RF: Radio frequency;
RMSE: Root mean square error; UHF: Ultra high frequency; VHF: Very high frequency

\section{Funding \\ This research project was financial supported by National Research Council of Thailand through the 2018 Graduate Research Scholarships.}

Availability of data and materials

Additional file: simulation and experimental results.

\section{Authors' contributions}

NS is the Ph.D. candidate who performed all the work in this paper. She is the main writer of this paper. PW is the main research supervisor of NS who helped her in fine-tuning the proposed scheme. All authors proposed the main idea, read and approved the final manuscript.

\section{Authors' information}

Nattakarn Shutimarrungson received the M.Sc. degree in Information Technology Management from Mahidol University, Bangkok, Thailand in 2004. She is currently a Ph.D. candidate in information

technology, Department of Information Technology, at Faculty of Information Technology, King Mongkut's University of Technology North Bangkok (KMUTNB), Thailand. Her research interests include wireless sensor networks and propagation models.

Pongpisit Wuttidittachotti is currently an associate professor and head of Department of Data Communication and Networking, Faculty of Information Technology at the Faculty of Information Technology, King Mongkut's University of Technology North Bangkok (KMUTNB), Thailand. He received his Master of Science in Information Technology from KMUTNB, in 2003. He obtained a scholarship to study in France and then received a Master of Research and Ph.D. in Networks, Telecommunications, Systems and Architectures from INPT-ENSEEIHT, in 2005 and 2009 respectively. Also, he was awarded a Postdoctoral scholarship from University of Paris XI in 2009. His research interests include eHealth/mHealth, MANET, information security, $3 \mathrm{G} / 4 \mathrm{G} / 5 \mathrm{G}$, networks performance evaluation, VolP quality measurement and QoE/QOS.

\section{Competing interests}

The authors declare that they have no competing interests.

\section{Publisher's Note}

Springer Nature remains neutral with regard to jurisdictional claims in published maps and institutional affiliations.

Received: 8 June 2018 Accepted: 19 March 2019

Published online: 11 April 2019

\section{References}

1. M.V. Ramesh, V.P. Rangan, Data reduction and energy sustenance in multisensor networks for landslide monitoring. IEEE Sensors J. 14(5), 1555$1563(2014)$

2. O. HJ, S. Lee, W. Chotikasathien, C.H. Kim, J.H. Kwon, Predictive landslide susceptibility mapping using spatial information in the Pechabun area of Thailand. Environ Geol 57(3), 641 (2009)

3. S. Biansoongnern, B. Plungkang, S. Susuk, Development of low cost vibration sensor network for early warning system of landslides. Energy Procedia 89, 417-420 (2016)

4. M.V. Ramesh, in IEEE, SENSORCOMM'09. Third International Conference on Sensor Technologies and Applications, 405-409. Real-time wireless sensor network for landslide detection (2009)

5. G.R. Teja, V.K.R. Harish, D.N.M. Khan, R.B. Krishna, R. Singh, S. Chaudhary, in 2014 IEEE International Advance Computing Conference (IACC). Land slide detection and monitoring system using wireless sensor networks (wsn) (2014), pp. 149-154

6. A. Terzis, A. Anandarajah, K. Moore, I. Wang, in Proceedings of the 5th ACM International Conference on Information Processing in Sensor Networks. Slip surface localization in wireless sensor networks for landslide prediction (2006), pp. 109-116

7. Y. Wang, Z. Liu, D. Wang, Y. Li, J. Yan, Anomaly detection and visual perception for landslide monitoring based on a heterogeneous sensor network. IEEE Sensors J. 17(13), 4248-4257 (2017) 
8. F. Wang, J. Liu, L. Sun, Ambient data collection with wireless sensor networks. EURASIP J. Wirel. Commun. Netw. (2010). https://doi.org/10.1155/ 2010/698951

9. P.R. Casey, K.E. Tepe, N. Kar, Design and implementation of a testbed for IEEE 802.15.4 (Zigbee) performance measurements. EURASIP J Wirel Commun Netw 23 (2010). https://doi.org/10.1155/2010/103406

10. A. Giorgetti, M. Lucchi, E. Tavelli, M. Barla, G. Gigli, N. Casagli, M. Chiani, D. Dardari, A robust wireless sensor network for landslide risk analysis: system design, deployment, and field testing. IEEE Sensors J. 16(16), 6374-6386 (2016)

11. H.C. Lee, K.H. Ke, Y.M. Fang, B.J. Lee, T.C. Chan, Open-source wireless sensor system for long-term monitoring of slope movement. IEEE Trans Instrumentation and Measurement 66(4), 767-776 (2017)

12. M.A. Weissberger, An initial critical summary of models for predicting the attenuation of radio waves by trees (Electromagnetic compatibility analysis center, Annapolis, MD, 1981) ECAC-TR-81-101

13. CCIR, Influences of terrain irregularities and vegetation on troposphere propagation. Geneva, Switzerland, pp. 235-236. CCIR Rep. (1986)

14. A. Seville, K.H. Craig, Semi-empirical model for millimetre-wave vegetation attenuation rates. Electron. Lett. 31(17), 1507-1508 (1995)

15. M.O. Al-Nuaimi, R.B.L. Stephens, Measurements and prediction model optimization for signal attenuation in vegetation media at centimetre wave frequencies. IEE Proceedings-Microwaves, Antennas and Propagation. 145(3), 201-206 (1998)

16. COST 235, Radio Propagation Effects on Next Generation Fixed-Service Terrestrial Telecommunication Systems. Luxembourg. Final Rep. (1996)

17. H.Y. Chen, Y.Y. Kuo, Calculation of radio loss in forest environments by an empirical formula. Microw. Opt. Technol. Lett. 31(6), 474-480 (2001)

18. J. Liang, Q. Liang, S.W. Samn, A propagation environment modeling in foliage. EURASIP J. Wirel. Commun. Netw. (2010). https://doi.org/10.1155/ 2010/873070

19. T. Tamir, On radio-wave propagation in forest environments. IEEE Trans. Antennas Propag. 15(6), 806-817 (1967)

20. T. Tamir, Radio wave propagation along mixed paths in forest environments. IEEE Trans. Antennas Propag. 25(4), 471-477 (1977)

21. D. TR Rao, N.T. Balachander, M.V.S.N. Prasad, Ultra-high frequency nearground short-range propagation measurements in forest and plantation environments for wireless sensor networks. IET Wireless Sensor Systems 3(1), 80-84 (2013)

22. R.M.D. Islam, Y.A. Abdulrahman, T.A. Rahman, An improved ITU-R rain attenuation prediction model over terrestrial microwave links in tropical region. EURASIP J Wirel Commun Netw 189 (2012)

23. J.D. Parsons, The mobile radio propagation channel (Wiley, 2000)

24. Y.S. Meng, Y.H. Lee, B.C. Ng, Empirical near ground path loss modeling in a forest at VHF and UHF bands. IEEE Trans. Antennas Propag. 57(5), 14611468 (2009)

25. T.S. Rappaport, Wireless Communications: Principles and Practice (Vol. 2) (New Jersey, prentice hall PTR, 1996)

\section{Submit your manuscript to a SpringerOpen ${ }^{\circ}$ journal and benefit from:}

- Convenient online submission

- Rigorous peer review

- Open access: articles freely available online

High visibility within the field

- Retaining the copyright to your article

Submit your next manuscript at $\boldsymbol{\nabla}$ springeropen.com 